\title{
Protection par Bobines d'Extinction à dissonance contre les mises accidentelles à la terre.
}

I a protection contre les mises à la terre en général et l'utilisation des bobines d'extinction à dissonance en particulier ont été traitées déjà dans deux articles de la revue $\mathrm{BBC}$ :

"Protection des réseaux à haute tension contre les effets d'une répartition asymétrique des capacités relatives à la terre " (1) et

"Protection contre les mises accidentelles à la terre" (2).

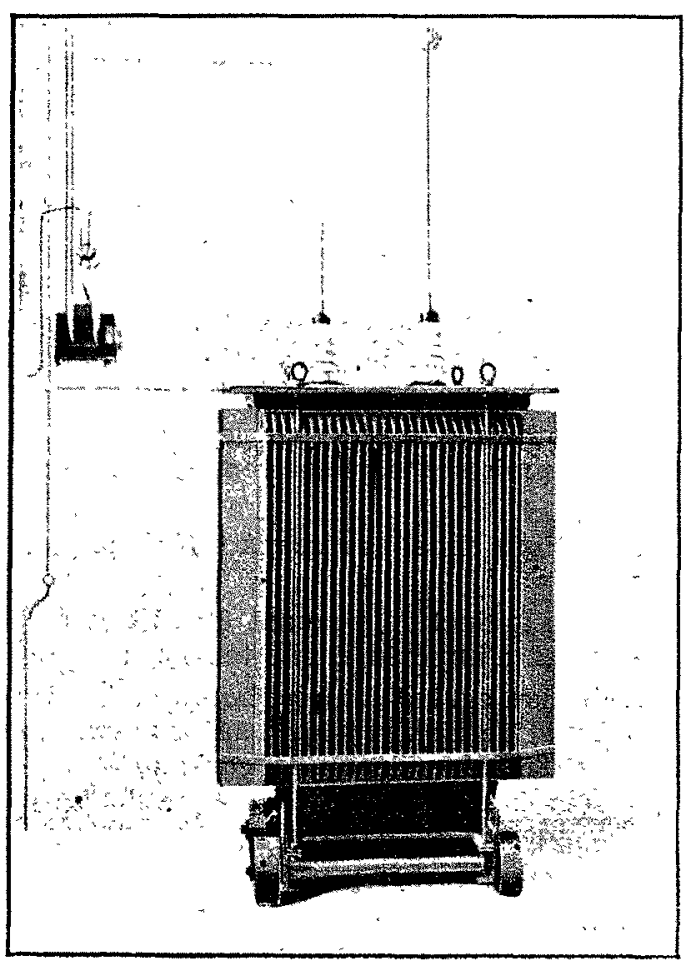

lijg, 1. - Bobine d'extinction à dissonance insérće dảns un réseau à $50 \mathrm{kw}$.

La question théorique et expérimentale ayant ćté traitée en détail dans ces articles, nous n'y reviendrons pas et nous résumerons simplement dans ce qui suit, les suites des mises à la terre, l'effet de la bobine d'extinction système $\mathrm{BBC}$ et ses avantages.

Nous avons été amenés à publier ce résumé par le grand nombre de demandes qui nous sont parvenues de nos lecteurs.

Les perturbations dans les réseaux électriques sont pour la plupart causćes par la mise accidentelle à la terre des lignes aćriennes et par les surtensions qui en résultent.

La Société An. Brown-Boveri et $\mathrm{C}^{\circ} \mathrm{a}$, pour cette raison, attaché la plus grande importance à la protection contre ces nises à la terre et peut proposer un dispositif de protection des plus sûrs (date de priorité du brevet suisse No 82.520 du 23-12-18). Ce dispositif consiste en une bobine de réactance à caractéristiques approprićes (voir figure 1) insćrće entre le neutre des installations et la terre.

Les avaries causéès par les mises à la terre et qui peuvent être, pour ln plus grande partie, éliminces par ce dispositil sont les suivanles :

10 Pour peu que l'intensité de l'are soil de quelques ampères,

(1) Voir Revue $B B C$, aônt 1920 , p. 202.

(2) Voir Revue BBC; juin 1921, p. 128: Article published by SHF and available il peut alteindre des longueurs considérables, d'où son extension sur les phases saines. Il provoque alors un court-circuit produisant unc perturbation de service qui, dans les cas les moins défavorables, dure quclques minutes, mais qui, le plus souvent, par suite des détériorations causées par l'arc de court-circuit lui-même, est beaucoup plus longue;

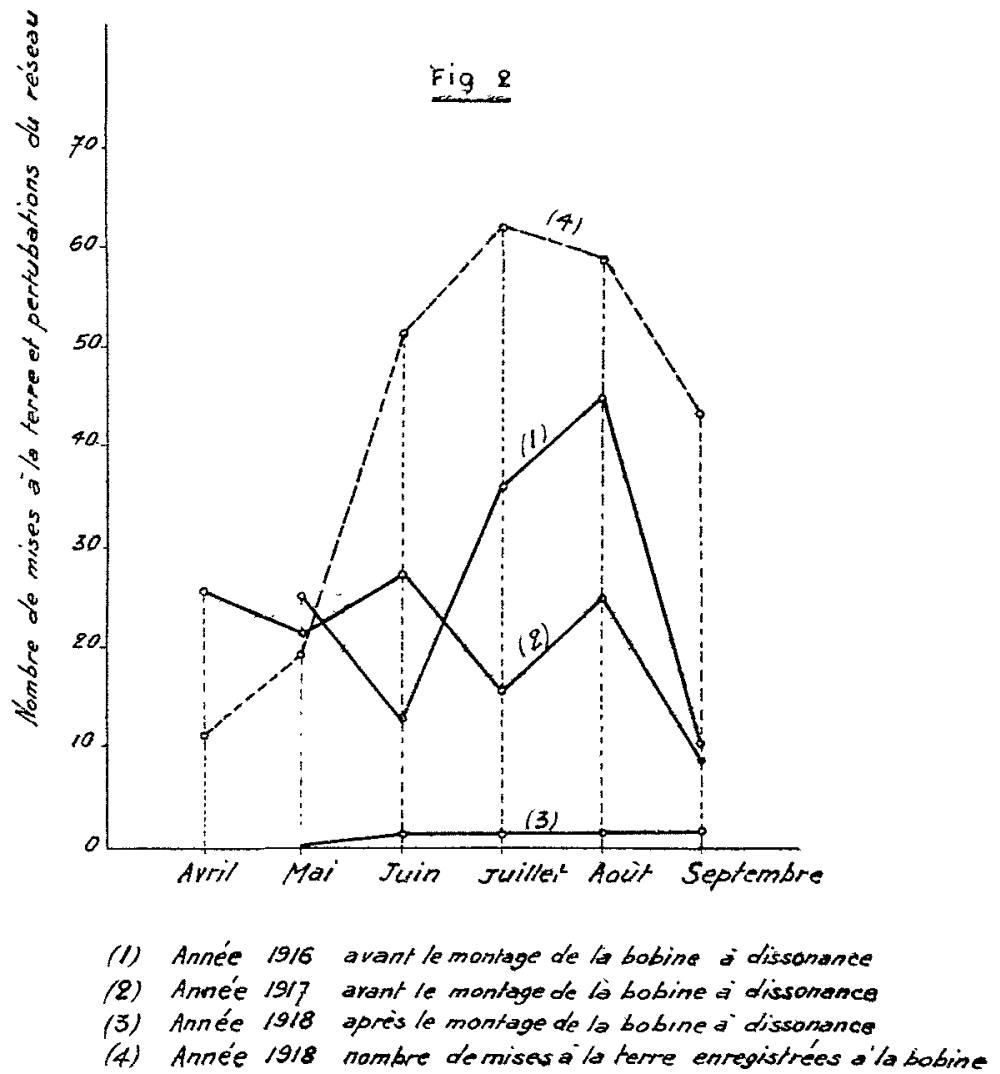

Fig. 2. - Centrale de Laufenburg. Longueur de la ligne $2 \times 134$ kilomètres. Tension de service 50.000 volts. Nombre de déclenchements automatiques par mois.

$2^{\circ}$ Surtensions par rapport à la terre et mises à la terre doubles. Les mises à la terre intermittentes provoquent des surtensions par rapport à la terre et créent, dans les phases saines des réseaux non protégés, des surtensions pouvant atteindre deux ou trois fois la valeur de la tension composée. Ces surtensions, à leur tour, provoquent de nouvelles mises à la terre sur ces phases. Les perturbations d'exploitation, de plus ou moins longues durées, se répercuteront alors sur les parties du réseau les plus éloignées. Les mises à la terre doubles sont en particulier à redouter, car elles produisent une chute de tension très dangereuse aux deux points de mise à la terre ;

$3^{\circ}$ Ondes à front raide. Dans le cas de mises à la terre intermittentes, il se produit, à chaque demi-période, un nouvel amorçage de l'arc à l'endroit de mise à la terre. A chaque amorģage, la tension de la ligne, au point considéré, diminue brusquement d'une valeur multiple de la tension composée jusqu’à zéro. Cette décharge se propage sous forme d'ondes progressives dans les deux directions de la ligne. Si ces ondes rencontrent des enroulements soit de transformateurs, soit de génératrices, elles créent des tensions dangereuses entre les différentes spires ou bobines;

$4^{\circ}$ Accroissement par rapport à la terre de la tension des phases saines jusqu'à la valeur de la tension composéc. Il n'existe pour 
ainsi dire pas de moyen propre à protéger les réseaux contre ces accroissements de tension. L'isolation d'un réseau bien comprise, devrait pouvoir supporter sans autre la tension composée.

La bobine d'extinction à dissonance permet d'obtenir l'extinction automatique de l'arc de mise à la terre. Elle élimine par conséquent les effets nuisibles de cet arc qui de plus ne peut s'allonger avant de s'éteindre. On arrive donc à supprimer simultanément des arrêts d'exploitation courts et longs à moins qu'il y ait destruction préalable du matériel isolant. Les bobines d'extinction suppriment aussi tout nouvel amorçage de l'arc et évitent complètement la production des ondes à front raide et des surtensions par rapport à la terre. (Voir résultats figure $n^{\circ} 2$ ).

La construction des bobines d'extinction est semblable à celle des transformateurs à bain d'huile. On admet en général pour les calculer qu'elles devront pouvoir être chargées sans interruption pendant une heure.

Afin de tenir compte des modifications pouvant se présenter dans la longueur des réseaux ainsi que de leur extension éventuelle, les bobines peuvent être construites avec plusieurs prises. En général, l'utilisation de plusieurs bobines de petites dimensions en lieu et place d'une seule de grandes dimensions est avantageuse. Chaque partie importante du réseau, qui peut être en exploitation pour elle-même, est munie alors de sa bobine d'extinction propre. Une solution particulièrement judicieuse consiste à employer dans le réseau tout entier des bobines du même type et de la même puissance. (Les avantages qui en découlent sont la réduction de prix, l'interchangeabilité et une plus grande facilité de répartition).

La bobine d'extinction à dissonance, système $B B C$, est dimensionnée de façon que le courant de mise à la terre ne soit compensé que partiellement. Ce dimensionnement est nécessité par l'existence dans les réseaux asymétriques (c'est-à-dire les réseaux dont les capacités partielles par rapport à la terre ne sont pas les mêmes), en régime normal, d'une tension entre le point neutre et la terre (tension d'asymétrie). Cette tension peut provoquer, dans le cas d'une compensation complète du courant de mise à la terre, des tensions de résonance (Voir revue $\mathrm{BBC}$, août 1920). La suppression de ces tensions de résonance est un des avantages principaux de la bobine d'extinction à dissonance.

Des essais minutieux ont montré que, même en choisissant pour le calcul de la bobine un courant différant sensiblement du courant de mise à la terre, la faculté d'extinction n'est pas influencée fortement (voir Revue $B B C$, juin 1921).

La bobine d'extinction à dissonance présente encore des avantages particuliers, principalement dans les réseaux aćriens, par rapport aux autres dispositifs de mise à la terre, tels par exemple qu'une mise à la terre du point neutre (directement ou par l'intermédiaire d'une résistance) ou des parafoudres à cornes avec résistances en sćrie.

Les parafoudres à cornes insérés en nombre suffisant sont capables de limiter les surtensions provoquées par les terres intermittentes. Par contre, ils ne peuvent éviter la formation d'arcs aux endroits avariés et n'ont pas d'influence, par conséquent, sur les perturbations d'exploitation qui en découlent. Un autre inconvénient des parafoudires à cornes provient de leur grand encombrement.

La mise à la terre du point neutre, par l'intermédiaire d'une résistance, amortit les surtensions provoquées par les terres.Elle fait, ainsi qu'une mise à la terre directe, déclancher, conformément au réglage des relais, les interrupteurs de la ligne endommagée et évite ainsi dans la plupart des cas, la formation de l'arc de courtcircuit. La mise à la terre du point neutre provoque áussi le déclenchement de l'interrupteur de la ligne lors de mises à la terre passagères. Ce déclenchement, en particulier dans les réscaux aériens, n'est pas à souhaiter. Une augmentation du courant au point de mise à la terre est aussi la conséquence de la mise à la terre du point neutre, d'où, l'accroissement. de la chute de tension au point avarié (danger pour le persomnel). Il est évident que la mise à la terre du point neutre exige l'insertion de relais dans toutes les phases.

La bobine d'extinction à dissonance est insérée entre le point neutre des installations el la terre, ce qui suppose au préalable que le ou les transiormateurs sont connectés en étoile. Lors du branchement de la bobine d'extinction sur un transformateur de puissance, il faut tenir compte de l'augmentation de charge que les enroulements du transformateur devront supporter ; en outre, par suite de la forte chute de tension dans ces enroulements, le pouvoir extincteur de la bobine peut être diminué. Il est bon de prévoir avec la bobine d'extinction un ampèremètre (éventuellement enregistreur) et un dispositif d'alarme renseignant le persomnel sur la mise à la terre accidentelle.

En resumé, les avantages des bobines d'extinction sont les suivants :

$1^{0}$ Extinction de l'arc de mise à la lerre dès son amorcage ;

$2^{\circ}$ Flimination des surtensions provoquées par les terres intermiltentes et des ondes progressives qu'elles créent ;

$3^{\circ}$ La tension d'asymétrie qui apparait entre la terre et le point neutre des réseaux, dont les capacités partielles sont inégales, ne produit pas, en service normal, de tensions de résonance lors de l'insertion de bobines d'extinction;

4o La continuité de l'exploilation est assurée en cas de terres passagères ;

$5^{\circ}$ Les bobines d'extinction écoulent les charges statiques ; les parafoudres à jet d'eau ou les bobines de self utiliscés spicialement (lans ce but peuvent donc être supprimés ;

$6^{\circ}$ Les pertes continues créées par les bobines sont minimes, car elles ne sont soumises à la pleine tension de phase que lors de mises à la terre ;

$7^{\circ}$ Des perturbations dans les réseaux téléphoniques ne sont pas à craindre;

So Les bobines d'extinction peuvent être utilisées pour la protection de réseaux à n'importe quelle tension;

$9^{\circ}$ Lcur installation est très simple, bon marché et d'une grancle sécurité de service. Elle n'exige aucun autre appareil qu'un ampiremètre avec son transformateur de courant et un sectionneur. L,isolation des bobines n'est à prévoir que pour la lension de phase;

$10^{\circ}$ Leur encombrement est faible, surtout en comparaison de celui des parafoudres à cornes ;

$11^{\circ}$ Dans les réseaux trìs ctendus el à basse tension de service, les bohines sont de faibles dimensions;

L'indication de la longueur du réseau el de la tension de service suffisent pour l'établissement de propositions provisoires. Pour des calculs exacts, les renseignements suivants sont nécessaires :

$1^{0}$ Longueurs simples de toutes les lignes du réseau ;

$2^{\circ}$ Sections de conducteurs et des fils de terre s'il en existe ;

$3^{\circ}$ Disposition des conducteurs et du fil de terre en tête du pylòne, distance entre conducteurs el entre conducteurs et terre ; $4^{\circ}$ Fréquence ;

$5^{\circ}$ Dimension et couplage des transformateurs et des génćratrices; $6^{\circ}$ Le point neutre des génératrices ou des transformateurs est-i sorti, sinon, est-il possible de le sortir?

$7^{\circ}$ Capacité des condensateurs s'il en existe.

S. V.

\section{Le transformateur d'essais triphasé à 1 million de volts de la G. E. C.}

La tension d'un millions de volts réalisée entre une branche du monophasé en étoile et la terre, relié à un point neutre de l'enroulement, a été avancée jusqu'à 1.200 et 1.500 .000 volts avec constatation d'une étincelle de 3 mètres de longueur pour la tension initiale, et de plus de 4 mètres pour un million et demi de volts. Cette étude, qui comporte nombre d'intéressants clichés de décharge (mono et triphasée - en triangle et en étoile), constitue la suite d'une précédente, parue en décembre dernier.
Après nous avoir initié aux expériences déjà lenlées - ou en cours - avec ce matériel à 1 million de volts à l'usine de la G. E. C. de Pittsfull, M. I.iston expose les condilions dans lesquelles, à son avis, semblable matériel peut être ulilisé pour les essais du matériel industriel. J. B.

General :lectric Review, décembre 1929, 11 février 1923. Génie Civil, 28 avril 1923. 


\section{Les fours électriques à induction à haute tréquence.}

Exposant le résultat de ses propres recherches et expériences, l'auteur de cette intéressante relation démontre qu'il est parfaitement possible de faire abstraction de tout noyau de fer dans les fours à induction si on peut faire appel à une fréquence assez élevée. Je verre $\mathrm{el}$ le quartz ne faisant pas obstacle à l'application de la Chaleur par induction, la bobine inductrice peut évidemment chre siluée à l'extérieur du récipient contenant le matériel à fondre. On peut done choisir l'atmosphère qui convient le mieux à l'opé- ration et provoquer les réactions chimiques avec toutes les précisions désirables, précisément grâce à ce choix.

Une des grandes applications de ces nouveaux procédés est la cuisson des tubes à vide sans le bombardement électronique généralement employé, quoique procurant de sérieux déchets dans la fabrication.

\section{Locomotive électrique triphasée de $3.000 \mathrm{HP}$.}

Ricemment mise en service par les FF. SS., cette machine est une des plus puissantes d'Europe. Le poids, entièrement utilisé pour l'adhérence est de 75 lonnes réparties sur cing essieux couplís it des rones motrices de $1070 \mathrm{~m} / \mathrm{m}$ de diamètre.

L'épuipement comporte deux moteurs identicies de 1.100 H.P. chacun, alimentés directement de la ligne de contact - 3.000 volts, $162 / 3 \sim$ - et qui transmettent le mouvement à l'essicu central au moyen d'une bielle triangulaire (les autres essicux sont accouples par lielles ordinaires). Je montage des moteurs ctant commutable à 8 et 12 pôles, qui peuvent être insérés en parallèle ou en casca de on en peut obtenir quatre vitesses de régime. I a vitesse maximum peut atteindre $50 \mathrm{~K}$ wh et l'effort de traction 10 à 16 tonnes, selon la vitesse.

En outre des moteurs, la ligne aérienne alimente deux transformatęurs de $12 \mathrm{KVA}$ réduisant la tension à 100 volts pour l'éclairage et les services divers : groupes de ventilation, groupe de compression, chaudière de thermo-siphon, etc. J. B. Industria, V. XXXVII, No

\section{Précautions à prendre pour éviter les perturbations dues à la mauvaise disposition des balais dans les machines électriques.}

Interessante collection de conseils el d'indication d'ordre prafique sur la façon d'éviter les ennuis généralement dus à la mauvaise disposition des balais, parmi laquelle une quinzaine de cas particuliers (les plus répandus) sont analysés successivement. Ce soml, en particulier, lorsque les balais ne sont pas dans la ligne neutre (court-circuit et itincelles echauffant le collecteur) ; lorsqu'ils ne sont pas équidistants ; lorsque, trop épais, ils recouvrent trop le lames ; lorsque la pression sur eux-mêmes est trop élevée ou frop fable (usure excessive ou noircissement du collecteur) ; lorsrü leur pression est inégale (inégale répartition de la charge et étincelles) ; lorsqu’ils ne coulissent pas dans leur gaine ; qu'ils ont trop de jeu (étincelles, échauffement, recuit du ressort) ; lorsqu'ils présentent, par rapport au rayon du collecteur aboutissant au point de contact, un angle défectueux ; lorsque la surface de contact des balais par rapport à celle du collecteur est trop élevée ; enfin, lors que les connections à chaque extrémité du shunt, sont défeclueuses (déperdition du courant par la gaine - détrempe du ressort).

Chaque cas est étudić en particulier et les remèdes pratiques sont exposés à côtó des camcléristiques des perturbations qu'ils entrainent.

\section{Accumulateurs à l'Hydrate de Plomb.}

Ta seule différence apparente entre ces accumulateurs et ceux du lype ordinaire réside dans l'épaisseur des plaques, qui est beaucoup plus grancle ; l'électrolyte est également beaucoup plus dense. La résistance intérieure est très petite et la capacité peut atteindre de trois à huit fois celle des types ordinąires ; la pureté chimique est également très supérieure ; collin, ils sont indestructibles ef peuvent rester chargés pendant des années sans détérioration.

En raison de la porosité de lhydrafe de plomb, l'électrolyte pénètre lacilement dans toute la placpue, doù rapidité pour la charge et la décharge. La première s'opère généralement sous un régime queleonque, mais préférablement, toutelois, avec une intensité égale au courant de décharge horaire. I.e rendement en énergie atteint alors $61 \%$ el avec la décharge en 12 heures jusqu'à $77 \%$

L'aut eur ajoute qu'expérimentalement un étément à l'hydrate de plomb a pu être chargé plus de cinq mille fois à une intensité centuple de sa normale sans aucune trace de détérioration. Il ajoute que ces appareils sont très en faveur en Angleterre. J. B.

Electrical Review, décembre 1922.

\section{Nouveau procédé de construction des pylônes en béton armé par éléments montés d'avance et assemblés à pied d'œuvre.}

Bien connús sont les avanlages du béton armé par rapport au métal et au bois dans la construction des poteaux de lignes ; mais ces avantages deviennent, la plupart du temps, inconvénients entrainant l'impossibilité de la solution en béton, dès qu'il s'agit de pylònes an lieu de simples poteaux : le moulage à pied d'ceuvre se révélant trop souvent trop onćreux quand ce n'est pas impossible.

M. Maréchal propose de tourner la difficulté par l'adoption du procéclé - déjà úlilisé en construction civile - - du moulage en sírie, a l'avance, d'éléments susceptibles de constituer le pylone

par leur assemblage sur place.

A cet effet, les éléments qu'il propose sont uniquement des cornières, dont l'assemblage diagonal, 2 à 2 , laisse entre elles un vide que l'on remplit de béton après y avoir disposé les armatures nécessaires, au fur et à mesure du montage. Entretoises et croisillons s'emboîtent dans des logements en saillie ménagés le long des montants et dans lesquels ils sont simplement scellés au ciment.

Ajoutons que ce genre de pylônes convient parfaitement pour les charpentes des stations de transformation en plein air. J. B. Revue Générale d'Electricilé, 23 décembre 1922.

\section{La Centrale thermique de Comines.}

Intéressante description de l'usine construite par l'Energic Electrique du Nord, au bord de la Lys, pothr servir de secours à celle de Wasquehal.

La plateforme de l'usine n'est qu'à 1 mètre au-dessus des plus hautes caux connues; le plancher des chaudières est à $5 \mathrm{~m}$. an-dlessus de ce niveau. Ia chaufferie, de $62 \mathrm{~m} .50$ de long, comporte dix chaudières Babcok el Wilcox du type marin, de 1.000 mètres de surface de chaulfe chacune, limbrées à 21 kilogs, et susceptibles de fournir en -marche normale 30.000 kilogs de vapeur à l'heure $(40.000$ kilogs en marche forcéc), dont huit sont munis de grilles mécaniques el-les autres, équipécs pour la chaulfe au charbon pulvérisé, d'économiseurs Green.

La salle des machines $(64 \times 24 \times 25,40)$ comporte trois groupes constitués chacun par unc turbine à action Schneider-Zoelly admetlant la vapeur à 18 kilogs effectifs par centimètre carré, surchauffée à $350^{\circ} \mathrm{C}$, et tournant à 1.500 tours minute. La réfrigération des alternateurs a lieu par circulation d'air frais. En outre, une turbine Rateau, de $3.500 \mathrm{KWS}$, a 3.000 tours minute, entraine un alternateur triphasé - constituant groupe auxiliairepour les clifférents services intérieurs (éclairage, pompes, pont roulant). 


\section{Procédés de construction des massifs de fondation des turbo-générateurs.}

On sait que la principale des caractéristiques à réaliser pour les fondations des turbo-générateurs est d'éviter les vibrations et les déformations sans entraver en rien l'entretien des organes accessoires. Les trois procédés de construction auxquels il a été fait appel jusqu'à ce jour (béton, métal et fondation mixte) ont été comparés dans un rapport présenté dernièrement à l'Am. Society of Civ. Eng. " en se basant surtout sur l'aptitude de chacun de ces procédés à réaliser les conditions ci-dessus plutòt que sur l'économie réalisable avec l'un quelconque d'entre eux (dont le maximum est de l'ordre de $5 \%$ seulement du prix total des fondations).

De cette comparaison résulte que le type de fondation mixte, avec un encombrement moindre, présente -- au point de rue des vibrations - sensiblement les mêmes avantages que la fondation en béton : Il y a toutefois lieu de tenir compte de la relativité de la période propre de vibration des fondations et de celle de la tul: bine. Enfin, le type de condenseur est de nature à influencer te choix du type de fondation : les condenseurs à injection nécessilant des fondations moindres que ceux à surface.

L'étude se termine par un graphique indiquant, pour le béton armé et le métal, le prix de revient des fondations parkW de puissance el la proportion du lomnage des fondation à celui des engins.

J. 13.

Potwer, 23 janvier 1923.

\section{Sous-station automatique à courant alternatif.}

Cette sous-station vient d'être récemment installée à KingsburgBoulevard (Saint-Louis) (Etats-Unis). L'auteur en expose les caractéristiques saillantes par rapport à la station génératrice et aux sous-stations voisines.

La station génératrice produit du courant à 13.200 volts, 60 périodes - distribué souterrainement aux sous-stations parmi lesquelles les sous-stations Enright et Kinsburg le transforment en 4.500 volts, 60 périodes, alors que la sous-station Page-Ho assure la même transformation et utilise comme secours, le réseau local, e: 25 périodes, par l'intermédiaire d'un translormateur de fréquence de $5.000 \mathrm{KW}, 4.500$ volts.
Tous les transfos et régulateurs ì huile sont munis de thermostats plongeant dans l'huile et actionnant les relais d'ouverture des interrupteurs lorsque la température dépasse $85^{\circ}$ C. Les leeders to départ, à 4.500 volts, sont munis de disjoncteurs à fermeture automatique. Enfin, par surcroîl de précaution, l'ouverture el li fermeture des robinets d'admission d'eau (refroidissement des transfos et régulateurs) sont commandés par des thermostats entre les limites de 65 à $75^{\circ}$ pour les transios et de 45 à 65 pour les régulateurs.

Electrical Horld, 11 octobre 1922

\section{Les interrupteurs fonctionnant en espace clos.}

Dans cet intéressant article, l'auteur procède à une étude objective très serrée de ces appareils et met en relief les conditions auxquelles, seules, leur fonctionnement apparaît sûr, desquelles il y a lieu de tenir grand compte pour établir une comparaison avec les autres types d'appareils. Sans jamais oublier que le fonctionnement en vase clos a pour effet d'introduire certains risques dans l'exploitation : un interrupteur est quelquefois supérieur aux engins modernes, surtout gand ces derniers ne sont pas rigoureusement adaptés aux conditions de travail de l'exploitation, étant donné limportance que prennent, dans le choix du type d'appariel, la robustesse des organes, la facilité de rupture, et les facilités d'entretion.

J. B.

\section{Commande automatique à distance de la Centrale hydraulique de Fairburg.}

Ce nouveau système de commande vient d'être récemment substitué à une commande funiculaire qui, malgré les perfectionnements progressivement apportés à ses guidages, ne donnait pas satisfaction, et permettait rarement une fermeture assez complète des vannes pour assurer un arrêt complet des turbines. Ln remaniement des conditions d'exploitation du réseau ayant conduit a une récente extension de la Centrale, on en profita pour substituer à cetle commande funiculaire une commande automatique dont l'auteur donne le détail de l'équipement actuel (constitué par deuß génératrices de $150 \mathrm{k} \mathrm{VW}$ (hacune). Celle nouvelle commande donne toute satisfaction : le démarrage et l'arrêt sont assurés sáns aucune main-d'œurre permanente, au moyen de flotteurs qui assirent ces opérations dans un ordre déterminć, et dont le bon fonctionnement est contrôlé périorliquement.

J. 3 .

I:leclricul World, 1/11 1922.

\section{Un Laboratoire à 1.000 .000 volts. $-1^{\text {re }}$ Partie.}

La Westinghouse Electric and Manufacturing Co vient d'installer un laboratoire d'essais à très haute tension. Avec l'emploi de teǹsions industrielles de plus en plus élevées, il devient nécessaire pour les laboratoires d'essais et de recherches de disposer de tensions de l'ordre de 1.000 kilovolts.

Le laboratoire est formé par un très grand hall de 3.5 mètres sur 40 mètres, et de 16 mètres de hauteur libre, il est desservi par un portique roulant et une voie de chemin de fer.

Le transformateur qui fournit directement la tension de 1.000.000 volts est du type à bobines cylindriques concentriques, les couches successives de l'enroulement forment condensateur de façon à uniformiser le champ électrostatique dans les isolants. L'article clécrit en détail les précautions nécessaires pour assurer la protection des bobines contre les décharges superficielles, et les ondes de surtension qui pourraient endommager les premières spires de l'enroulement.

Ja plus grosse difficulté à vaincre est celle de la sortie du fil conducteur à haute tension. Dans le transformateur de la Westinghouse, la borne de sortie est formée par l'enroulement, autour d'un tube d'acier de 15 centimètres de diamètre, de couches successives de papier imprégné et de feuilles de métal. L'ensemble pèse environ 12 à 13 tonnes.

Voici les principales caractéristiques du transformateur :
Nombre de bobines secondaires .... Nombre de spires du secondaire .... Longueur du fil secondaire.........

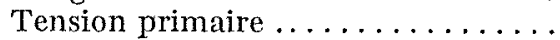
Tension secondaire............. Diamètre de la cuve............. Hauteur de la cuve............. Puissance apparente...........

31
36.000
$110 \mathrm{~km}$.
$5.000 \mathrm{volts}$
$1.000 .000-$
$.1 \mathrm{mètres}$
$5 \quad--$
$1.000 \mathrm{kva}$

Note. - On trouvera dans le journal A. I. E. E. (octobre 1922) et le General Electric Review (décembre 1922, février 1923) une description du laboratoire à haute tension de la General blectric ca La tension de 1.000.000 volts est obtenue par plusieurs transfor mateurs fonctionnant soit en étoile à 578.000 volts chacun, en triphasé, la tension étoilće étanl 1.000 .000 de volts, soil en sélé, au moyen d'un transformateur d'excilation intermédiaire (2.50 volts 2.500 volts) convenablement isolé.

Le systeme de la Westinghouse permettrait au contraird'alteindre 2.000 .000 de volts avec deux unités en séric. J. M. 


\section{Un Laboratoire à 1.000 .000 volts. $-2^{\text {me }}$ Partie.}

La mesure du potentiel se fait au moyen d'un éclateur à électrodes sphériques. Cette forme d'éclateur possède une précision remarquable, que les conditions atmosphériques affectent fort peu. Toutefois le diamètre des sphères doil être de l'ordre de grandeur de la longueur d'étincelle. Pour le transformateur à 1.000 .000 de volts, on dut construire en conséquence, un nouvel éclateur, avec des sphères de 1 mètre de diamètre. Ces sphères furent coulées en bronze et soigneusement tournées et rectifiées. Chacune, après finition, pesait près de 200 kilogs. L'une d'elle fut fixce au sommet de la borne de sortie du transformateur, lautre fut suspendue, all moyen d'une lige isolante, à une crémaillère, commandée par un moteur. Une échelle permet de lire très exactement l'écartenent des sphères, le repérage du zéro de l'échelle s'effectuant automatiquenent. La sphère mobile est reliće à la terre à travers des résistances limitant le courant à 1 ampère.

Je courant arrive au laboratoire à 22.000 volts. Un transformateur à prises variables et un régulateur d'induction permet de faire varier la tension d'alimentation du transformateur d'essais entre 0 et 5.000 volts d'une façon uniforme. Au cas où le courant a 22.000 volts ne serait pas suffisamment régulier, on peut employer un groupe moleur générateur de $600 \mathrm{kva}$. Tous ces appareils sont protigés par des disjoncteurs convenables. Par surcroît de précautions, on a installé une sorte de bouton-poussoir que l'opérateur pent mancuvrer avec le pied. Il suffit alors qu'on lâche cet interrupteur pour que le courant soit coupé.

L'éclateur à électrodes sphériques n'est pas toujours suflisant pour mesurer les hautes tensions. Il ne donne en effet une indication que si on lui fournit la puissance presque totale du transformateur, ce qui est inadmissible dans un essai. Or on ne peut se baser sur le rapport de transformation pour avoir une idce exacte de la tension secondaire. Les effets de capacité sont en effet tels. qu'ils provoquent à pleine charge une surtension de près de $25 \%$.

Dans le laboratoire de la Westinghouse on emploie un voltmètre spécial, dit voltmètre de crête d'onde basé sur la proportionnalité qui existe entre la valeur moyenne du courant de charge d'un condensateur et la valeur maxima de l'onde de tension qui provoque ce courant de charge. Comme condensateur, on emploie la borne de sortie précédemment décrite. Le courant de charge est redressé au moyen d'un redresseur mécanique (l'article explique pourquoi on a rejeté l'emploi de redresseurs à cathode incandescente), puis envoyé à un ampèremètre genre d'Arsonval à plusieurs sensibilités, étalonné directement en kilovolts.

Le laboratoire comprend aussi un transformateur à 500.000 volts, de construction semblable au précédent et muni des mêmes appareils de contrôle et de mesure. Les deux transformateurs peuvent être placés en série, ce qui fournit une différence de potentiel utilisable de 1.500 .000 volts.

On a prévu également un réservoir d'huile pouvant contenir plus de 200.000 litres d'huile afin de faire des essais d'appareils immergés dans l'huile. Ce réservoir d'essais est alimenté par tout un système de tuyauteries permettant de filtrer l'huile, de la chauffer ou de la renvoyer à deux réservoirs extérieurs au laboratoire qui emmagasinent l'huile à sa sortie des vagons spéciaux.

Enfin, à côté du laboratoire se trouve un terrain plat, permettant des essais en plein air.

J. M.

J.-F. Peters el D.-F. Hiners. - Electrical World, 14 avril 1923 , page $849,31 / 2$ pages, 5 figures.

\section{La Houille Blanche en Norvège.}

l.a Norvège est le pays du monde qui a la plus grande puissance hydraulique par tête d'habitant. On estime la puissance totale de ses chutes d'eau à 12.000 .000 de chevaux, dont 1.650 .000 sont actucllement utilisés.

L'article décrit les ressources hydro-électriques des diverses régions de la Norvège, ainsi que le caractère particulier des cours d'eau de chacune de ces régions. Le Sud, plus favorisé au point de vue géographique et économique est aussi la partie du pays la plus riche en chutes d'eau utilisables. Les principales installations sont rapidement passées en revue et leurs caractéristiques les plus importantes sont signalées.

L'énergie hydro-électrique de la Norvège est utilisée surtout par de grandes usines électrochimiques ou électrométallurgiques, et pour l'alimentation des chemins de fer électriques. Une bonne partie de la puissance hydraulique actuellement utilisée sert directement à faire marcher des scieries.

J. M.

Electrical World, 24 mars 1923, p. 677, 5 pages $1 / 2$, 6 figures.

\section{L'amélioration du facteur de puissance au moyen de condensateurs statiques.}

l'artlcle étudie d'abord la meilleure valeur à adopter pour la capacité du condensateur par cheval du moteur asynchrone dont on veut améliorer le facteur de puissance. L'auteur estime que pour les moteurs à cage d'écureuil, il convient de ne pas dépasser $11 \mathrm{~m}$. f. par cheval (sur courant diphasé, 220 volts, 60 périodes), de façon à ne pas avoir de courant capacitant aux faibles charges.

L'effot des harmoniques est renforcé par le condensateur, et les courants parasites provoqués par ce phénomène gênent les mesures et déforment la courbe du courant débité par le réseau. On peut s’en affranchir en partie en plaçant une réactance en série avec la capacité. Cette réactance produit aux bornes du condensaleur une surtension dont il faut tenir compte. D'autre part, cette réactance ne domine l'effet d'un harmonique donné qu'au dessus d'une certaine valeur (par rapport à la capacitance du condensateur employé) valeur qui dépend du rang de l'harmonique. L'auteur propose la valeur de 18 à 20 pour cent, et indique de plus les modifications à apporter à ses conclusions lorsqu'on a affaire à une autre tension ou à une fréquence différente.

Electrical World, 31 mars 1923 , page 739,2 pages $1 / 2$, 3 schémas.

\section{Quelques problèmes posés pour la construction des alternateurs à grande vitesse et leur solution.}

L'arlicle examine les principales dispositions constructives des allernateurs à grande vitesse Parsons et Metropolitan-Vickers.

Il décrit en particulier : les cables Parsons à conducteurs mulliples, destinés à éviter les courants de Foucault qui se produisent thans les barres pleines de grandes dimensions ; la forme à donner aux encoches pour éviter la production dharmoniques; les divers procédés de refroidissement, y compris le refroidissement par eau du rolor ; la cuestion de l'instabilité de l'excitatrice, et enfin les effets des courts-circuits sur la masse même du rotor.

Quand en effet un alternateur est mis en court-circuit, le champ résultant n'est plus rigoureusement fixe par rapport au rotor, et ces oscillations déterminent des courants intenses dans la masse de celui-ci. Ces courants tendent à se fermer par les supports métalliques des têtes de bobines. Si l'on ne prend pas des précautions spéciales pour assurer un bon contact entre ces pieces et le rotor, ou pour les isoler (ce qui esl plus difficile à réaliser pour les grandes vitesses périphériques), il se produit des arcs locaux qui endommagent les machines; L'auleur lournit des relevés d'oscillogra- phes à l'appui de ses assertions. La différence de potentiel entre les extrémités du rotor atteint 20 volts quand on coupe brusquement l'excitation. De pareilles tensions déterminent évidemment un échauffement intense de la masse du rotor.

Dans les discussions qui suivent l'article, on montre diverses installations de refroidissement par l'air en circuit fermé, et un procédé pour indiquer électriquement le degré d'humidité de l'air de refroidissement au moyen de deux détecteurs formés de deux pièces métalliques isolées au presspahn. L'un est protégé contre l'accès direct de l'humidité, et l'autre, au contraire, est placé directement dans le courant d'air. On détermine'le degré de l'humidité en mesurant la différence des résistances des détecteurs, le détecteur humide ayant naturellement une résistance d'isolement inférieure à celle du détecteur sec.

En ce qui concerne l'instabilité de l'excitatrice d'un alternateur, référence est faite, au cours de la discussion, au journal $A$. I. E. E. 1922, vol. 41, p. 731. J. M. J. Rosen, Journal I. E. E., vol. 61, av. 1923, p. 439 à476 


\section{Les installations électriques en Italie.}

D'après les stalistiques publices par le Ministère des Travaux publics, la puissance de toutes les installations hydroćlectriques d'Italie atteint $1.4 .49 .945 \mathrm{ch}$, soit une augmentation de $252.318 \mathrm{ch}$., par rapport à 1920. En 1915, la puissance totale était seulement de $960.000 \mathrm{ch}$. ; 67 installations nouvelles ont été faites en 19211922 ; 15 réservoirs d'une capacité de $78.375 .000 \mathrm{~m}^{3}$, ont été aménagés. On a commencé les travaux de 51 nouvelles usines, dont la puissance sera de 631.651 ch. el de 27 réservoirs d'une calpaciti de $209.659 .000 \mathrm{~m}: 3$. Le programme des socicitcis électriques est d'at* teindre, en 1928, une foree moyeme de $2.400 .000 \mathrm{ch}$.

Il faut ajouter aux installations hydroćlectriques les usines fonetionnant au charbon dont la puissance est de $500.000 \mathrm{ch}$. R. G. E., 21 mars 1923.

\section{L'Aménagement du Saint-Laurent.}

Des études sont entreprises par les Etats-Unis el le Canada visant l'aménagement du Saint-Laurent, tant au point de vue de la navigation qu'à celui de l'utilisation de l'énergie, entre le lac Ontario et Montréal.

Son débit régularisé serait de 6.000 mètres cubes à la seconde, et la chute totale sur le parcours considéré est de $65 \mathrm{~m}$. 50 . Il est à remarquer que celte chute est localisce en quelques points oì se trouvent des rapides de faibles longueurs.

Néanmoins, on objecte qu’il serait plus économique d'oblenir 2 millions de chevaux supplémentaires au Niagara. D'autre parl, il y aurait de nombreuses questions militaires et politigues à régler avant de commencer les travaux.

\section{BIBLIOGRAPHIE}

La Construction des grands barrages en Amérique, par W.-P. Creager, de la Société des Ingénieurs civils américains. Traduit de a'anglais par MM. Callandreau, ingénieur E. C. P., et Hunbert, ingénieur E. P. Z. - Un volume in-8 $(25 \times 1)$ de 243 pages, avec 88 figures et 7 planches. - Gauthiers-Villars et Cie, éditeurs, Paris, 1923. - En vente à la Librairie de la Houille Blanche, à Grenoble. Prix, broché, 25 francs.

En consultant cette traduction de l'ouvrage de M. Creager, les ingénieurs français pourront se rendre compte de la manière dont les ingénieurs américains établissent leurs projets de barrages.

Dans les deux premiers chapitres, on trouvera des indications sommaires sur la prospection et les recherches préliminaires au choix de l'emplacement de l'ouvrage à construire, ainsi que sur le choix du type de barrage à employer : terre, maçonnerie ordinaire, voûte ou béton armé.

Dans les chapitres III à V, l'auteur étudie les diverses forces auxquelles sont soumis les barrages - pression de l'eau sur les parements, choc des vagues et poussée éventuelle de la glace au sommet, poussée des vases à la base, sous-pressions le long de la fondation ou des joints horizontaux - et, par des considérations théoriques d'ailleurs assez élémentaires, en déduit six conditions de stabilité pour un barrage en maçonnerie du type plein à gravité.

Les chapitres VI et VII contiennent, à titre d'exemple, les calculs de projets de barrages de ce type pour des hauteurs de 30 et de 60 mètres. La détermination des efforts verticaux de compression y est d'ailleurs faite par la vieille méthode de la courbe des pressions, dans laquelle on a fait intervenir l'action des sous-pressions ainsí que celle de la poussée de la glace.

Le chapitre VIII est consacré aux barrages évidés en béton armé, à parements amont rectiligne ou courbe, tandis que le chapitre IX traite des barrages en voûte.

Enfin les chapitres X à XII, qui terminent l'ouvrage, contiennent quelques renseignements pratiques sur la préparation et la protection du sol de fondation, sur la composition des maçonneries et sur leur drainage, sur l'établissement des joints de contraction, ainsi que sur l'évacuation des crues par vannes, déversoirs, ou siphons automatiques.

Théorie de l'Allotropie, par le Dr Surts, professeur de chimie générale à l'Université d'Amsterdam. Traduite par J. Gillis, docteur en sciences chimiques. Un volume in- 8 raisin $(25 \times 16)$ de 524 pages et 239 figures. 1923. Gauthier-Villars, éditeur. 55 fr. En vente à la Librairie de la Houille Blanche, 23, Grande-Rue, Grenoble.

On sait les progrès immenses qui ont pu être réalisés dans l'étude scientifique des phénomènes les plus variés que l'on rencontre à chaque pas en chimie, électrochimie, électrométallurgie et métallurgie, grâce à la loi des phases de Willard-Gibbs.

Si on doit citer parmi les savants illustres qui contribuèrent à mettre en valeur les principes féconds du physicien américain :
Van der Waals, Vant'Hofl, Baklutis, Rozeboom, 11. Le Chatetier il ne faut pas oublier une pléiade d'expérimentaleurs habiles, Osmond, Charpy, Kotaro Honda, L. Guillet et bien d'autres plas modestes qui contribuèrent largement à développer les applications de la doctrine des phases. Toutefois parmi ceux qui onl poursuivi avec le ṕlus de succès l'étude théorique et expérimentale de cette doctrine, on doit retenir le nom de Smits qui éludia tout d'abord les phénomènes qui se manifestent dans les systèmes binaires et ternaires quand se présentent des phénomènes critiques en présence des corps solides. Dans les derniers temps, il a en pour but d'approfondir la connaissance des rapports qui existent entre les différentes phases d'un système unaire, en se basant sur les considérations théoriques acquises par l'étude des autres systèmes. Smits réussit de cette manière à découvrir des points de vue insoupçonnés qui ont révélé d'autre part l'existence d'un nouveau champ d'expérimentation.

C'est ce qui apparaîtra clairement en prenant connaissance de la Théorie de l'Allotropie de M. A. Smits. Les deux hypothèses qui la guident ont permis de grouper et d'expliquer un ensemble de phénomènes en apparence très disparates.

En électrochimie, nous ne saurions manquer d'attirer l'altention d'une part sur l'application de la théorie de Smits aux équilibres électromoteurs, d'autre part sur l'emploi des formules du potentiel expérimental qui sont absolument indépendantes de l'effet Volta et enfin sur les explications nouvelles des phénomènes de la pola. risation (cathodique et anodique), surtension, passivité, etc.

Aussi, attendait-on avec impatience la traduction de l'ouvrage du savant professeur de l'Université d'Amsterdam. Flle sera accueillie avec la faveur qu'elle mérite par les chimistes, les physiciens et les ingénieurs français, métallurgistes ou électrochimistes pour lesquels elle sera un guide et un auxiliaire des plus précieux.

$$
\text { P. DEJEAN. }
$$

\section{$\star \star \star$}

Fabrication de l'acier au four Martin, par A. BАнвњnot. - Un volume grand in-8 de 548 pages avec 160 ligures. - Encyclopédie minière et métallurgique, publice sous la direction de M. L. Guillet. - J.-B. Baillère et fils, éditeurs. - En vente à la Librairie de la Jouille Blanche, 23, Grande-Rue, Grenoble.

La lecture de cet excellent ouvrage est vérilablement tris attrayante. On sent en Ie parcourant qu'il a élé écrit par un praticien qui connaît à fond ce dont il parle. Aucune vieille rengaine et rien de suranné ; telle est la caractéristique de ce livre qui fait le plus grand honneur à celte encyclopédie minière et métallurgique, dont la qualité s'affirme chaque fois que paraît un nouvel ouvrage.

Après un historique très sobre mais suffisant pour montrer l'importance et le développement toujours croissanl de l'invention de Martin, l'auteur entre dans son sujet par une étude rapide, mais suffisante pour en donner une idée, des matériaux et malières premières employées dans les fours Marlin. Or, on sail le rôlọ 
important et complexe de ces matériaux en métallurgie. Ils doivent elre susceptibles de résister aux températures énormes des diverses parlies des fours, empêcher les déperditions trop grandes de chaleur ; servir à la récupération des chaleurs perdues et enfin, joter leur rôle dans les réactions du four.

M. Barberot aborde ensuite le chauffage des fours Martin. Après une étude approfondie du mode de chauflage le plus courant, e'est-ì-dire par le gaz des gazogènes (description des gazogènes molemes et de tous les appareils qu'entraine l'utilisation de ce gaz), il aborde l'étude des autres modes de chauffage dont (quelcues-uns deviennent de plus en plus à l'ordre du jour. Chauffage au naphle, au gaz de four à co'ie, au gaz naturel, au goudron et cufin atu charbon pulvérisé.

La construction des fours Martin est traite avec toute l'ampleur nécessaire et c'est peut-être là qu'on sent le mieux la maîtrise de l'auleur en pareille question. Les domnées y sont nettes et précises. On sent.qu'elles ont été rassemblées par un praticien qui a cu besoin de les uliliser et qui sait l'importance relative qu'on doit accorder à chacune d'elles.

liétude des opérations dans le lour Martin porte également la marque d'un aciériste consommé. Après l'étude générale du procédé et les méthodes classiques, un important paragraphe est consacré aux procédés spéciaux pour fabrications intensives.

Enfin, après nous avoir foumi tous les détails la coulée, les lingotieres et les lingots, l'auteur passe en revue les appareils de manutenlion et les dispositions générales d'aciéries Martin.

(et ouvrage intéressant el utile au premier chef se termine par l'élude des bilans calorifigues el des prix de revient.

\section{P. DEJEAX.}

Forgeage et Laminage, par 1. (iEvze. - - Ln rolume grand in- $S$ de 362 pages avec 229 figures. - Encyclopédie minière el mélallurgique publice sous la direction de M. L. Ginllet. - J.-B. Baillère et Fils, éditeurs. En vente à la Librairie de la Houille Blanche, 23, Grande-Rue, Grenoble.

On rencontre assez facilement de bons ouvrages relatifs au forgeage, encore que le forgeage des très grosses pièces soit souvent traité d'une manière très sommaire ; mais les ouvrages relatifs au laminage sont beaucoup plus rares. Il semble que les spécialistes lamineurs tiennent à garder jalousement pour eux les domnćes qu'ils ont péniblement amassées par leur pratique personnelle et on peut bien le dire aussi au prix de nombreux tàtonnements qui ont coûté souvent fort cher aux usines. Cette pratique de travail sous le boisseau a souvent conduit à des résultats déplorables. On s'en est aperçu surtout lorsqu'il s'est agi d'équiper électriquement les laminoirs. Lorsque les électriciens chargés de celte besogne demandaient les données indispensables pour étudier un matéricl approprić, ils ne pouvaient bien souvent rien obtenir parce que les données qu'ils demandaient n'avaient jamais été déterminces d'une manière scientifique. Quand à l'étude des cannelures de cylindres, de quel mystère n'était-elle pas entourée?

Aussi faul-il savoir gré à M. Geuze d'avoir cherché à jeter un peu de clarté sur toutes ces questions souvent fort obseures, où il reste encore beaucoup à ćtudier .

Dans la première partic, relative au forgeage, il passe en revue : les marteaux-pilons à simple et à double effet; les moutons à estamper à friction et à vapeur ; les presses à forger et les fours à chauffer les pièces.

La seconde partie, relative au laminage, débule par quelques renseignements utiles sur la fabrication du fer puddlé. Bien que celte fabrication tende à disparaître de plus en plus, ces données, ne serait-ce qu'au point de vue historique, sont loin d'être dépourvues d'intérêt. On y passe ensuite en revue tous les modes de laminage, avec la description détaillée des appareils utilisés : laminoir blooming, trains de laminoirs finisseurs réversibles, laminoir en trio de 650 millimètres, trains contimus avec cages en enfilade, laminoirs et fours a réchauffer pour la fabrication des plaques de blindage installés aux Wickers-Works, à Sheffield, làminoirs à tôles, laminoirs universel Kennedy, laminage à froid, laminoir à bangages Munton, grue-enclume el table de forge Kennedy, trains montés pour wagons, voitures et locomotives.

Cette énumération suffit à montrer l'utilité et l'intérêt d'un tel ouvrage.

P. DeJEAN.

\section{$+$}

La Houille Blanche, par H. CAvaithis, Professeur au Lycée de Bordeaux. Un volume in-16 de 216 pages. (Collection Armand Colin, 103, boulevard Saint-Michel, Paris. - Relié, 6 fr. ; Broché, 5 fr. - En vente à la Librairie Rey, Grenoble.

L'ouvrage de M. Cavaillès mérite de retenir l'attention de toutes les personnes qui s'intéressent à la houille blanche. L'étude géographique de la houille blanche que l'auteur nous présente est certainement le premier ouvrage renseignant le public sur ce qu'est la houille blanche, et présentant un tableau d'ensemble de l'état actuel de l'industrie hydro-électrique en France et dans le monde entier.

Ce petil ouvrage débute par l'historique et par les caractéristiques essentielles des chutes. Dans cette première partie composée de quatre chapitres, l'auteur traite : des eaux courantes, source d'énergie, de la production de l'énergie, de la distribution et de la vente de cette énergie, de l'utilisation du courant. Cette partie à l'usage de ceux qui ne sont pas techniciens situe d'une façon simple et claire l'industrie hydro-électrique.

La seconde partie intitulée "Les Pays de la Houille Blanche" est un tableau de la répartition de la houille blanche par régions naturelles; elle débute par l'étude de la France, en particulier les deux Savoies et le Dauphiné, berceaux de la houille blanche, puis les Alpes du Sud, les Pyrénées et le Massif Central, Cette seconde partie est très importante puisqu'elle prend à peu près la moitié du volume. Pour chacune des régions décrites, l'auteur présente un état des ressources en énergie hydraulique et de l'utilisation qui en est faite. Les données numériques ont été puisées aux sources les plus sûres, fournies par les statistiques officielles et par les entreprises industrielles.

L'avant-dernier chapitre intitulé "La Houille Blanche hors de France \%. Ce chapitre contient des renseignements très précieux parce qu'il contient des domnées qu'il est difficile de se procurer sans de longues recherches dans les ouvrages étrangers. L'auteur passe en revue la houille blanche en Espagne, en Italie, en Suisse, en Scandinavie, au Canada, aux Etats-Unis.

M. Raoul Blanchard, dans la remarquable bibliographie qu'il a faite de l'ouvrage de M. Cavaillès, dans la Revue de Géographie alpine (Tome XI, 1923), dit, à propos de ce chapitre : que M. Cavaillès nous a rendu là, pour l'étude de la Houille Blanche et pour la connaissance géographique de ces pays mêmes, le plus signalé service.

Enfin, le dernier chapitre, "La Houille blanche dans le monde ", est un véritable modèle.

$L$ 'ouvrage de $M$. Cavaillès est à recommander, non seulement aux géographes, mais à tous ceux qui s'intéressent au développement de l'avenir économique de notre pays, hommes' politiques, Ingénieurs, Industriels, et au grand public.

L'électrochimie et l'électrométallurgie, par Albert LEvassEuR. Ingénieur A. et M., professeur d'électrochimie et d'électrométallurgie à l'École d'Électricité et de Mécanique industrielles de Paris, et à l'Ecole Bréguet. 2 e édition, revue et augmentée. Un vol. in-8 de 267 pages avec 43 figures. Prix net : $16 \mathrm{fr}$. Dunod, éditeur, 47, quai des Grands-Augustins, Paris (VIe). En vente à la Librairie Rey, Grande-Rue, à Grenoble.

Parmi les branches de l'industrie qui se sont très largement développées pendant ces dernières années, il en est peu dont l'essor ait été aussi important, à la fois par lui-même et par ses répercussions techniques et économiques, que celui de l'électrochimie et de l'électrométallurgie. Or, les livres de langue française traitant ces questions, peuvent, pour la plupart, être rangés dans deux catégories. Les uns donnent une vue panoramique très étendue des diverses fabrications, avec une foule de détails descriptifs ; mais leur insuffisance pour ce qui est de la théorie et des principes scientifiques fondamentaux, ne permettrait pas au lecteur de remplir effectivement les fonctions d'ingénieur électrochimiste ou électrométallurgiste.

Les autres ont exclusivement pour objet la science pure. Ces traités ne sauraient convenir au technicien, car ils n'étudient ni fabrication, ni procédés, ni appareils.

L'ouvrage de M. Albert Levasseur comble la lacune que laissent subsister les deux catégories de livres dont nous venons de parler. Cet ouvrage destiné aux industriels, aux ingẻnieurs ou élèvesingénieurs, est la rédaction d'une partie du Cours d'électrochimie 
et delectrometallurgie que lauleur professe depuis plusicurs annés à l'Ecole d'Electricité et de Mécanicque industrielle de I'aris, et à l'Ecole Bréguet. L'auteur y donne une place prépondérante aux théories scientifiques fondamentales et aux directives générales $d$ 'application industrielle, faisant ainsi passer au second plan letude speciale des diverses fabrications.

M. I.evasseur estime, en effet, avec juste raison, que l'enseignement technique doit apporter autre chose qu'un amas de faits particuliers, et que son ròle capital est de fournir aux étudiants des principes et des méthodes leur permettant de résoudre dans les conditions les plus rationnelles, les problèmes scientifiques et pratiques qui, dans l'industrie, se posent constamment à l'ingénieur.

L'auteur rappelle dans la première partie de louvrage, les connaissances générales particulièrement importantes, telles que : thermodynamique, thermochimie, statique chimique, puis, dans la deuxième partie, traite de la théorie de l'électrolyse. La troisième partie est consacrée à l'électrochimie et à l'électrométallurgie industrielles par voie humide et enfin, la quatrième à l'électrochimie et l'électrométallurgie par voie sèche.

Cette nouvelle édition est la mise à jour de lexcellent ouvrage de M. Levasseur.

(S. V.)

\section{$*$}

Données numériques d'Electricité, magnétisme et électrochimie. Rédigées par MM. Boll (Paris); G.-I. Higson (Londres); M. MALAPERT (Paris) ; R.-E. SlAdE (Londres); G. V. Weisse (Lausanne). Préfacé du $\mathrm{D}^{\mathrm{r}}$ F.-B. Jevvett, Vice-Président de la WesternElectric $\mathrm{C}^{\circ}$ (New-York). - Un volume $(22 \times 28)$ contenant 114 pages de tableaux numériques. - Prix : Broché, 30 francs ; cartonné, 40 francs. - Secrétaire général : C. Marie, $D^{r}$ èssciences, 9, rue de Bagneux, Paris (VI $\left.{ }^{\mathrm{e}}\right)$. - En vente à la Librairie de la Houille Blanche, 23, Grande-Rue, Grenoble.

Ce volume constitue un tirage à part du volume IV des Tables annuelles de constantes et données numériques de chimie, de physique et de technologie.

Il contient les constantes et données numériçues relatives à l'électricité, au magnétisme et à l'électrochimie, parues pendant les années 1913 et 1916 inclues.

L'importance de ce fascicule montre le développement des recherches dans ce domaine. Le lecteur y trouvera tous les documents numériques parus dans les périodiques scientifiques et techniques du monde entier.

Chacun des chapitres a été rédigé par un spécialiste et les tableaux contenus dans ce volume permettent de voir quelles ont été, pendant ces années de 1913 à 1916, les questions qui ont été particulièrement étudiés.

Ainsi que le dit le $\mathrm{D}^{\mathrm{r}}$ Jewett dans sa préface, les phénomènes photo-électriques, thermo-ioniques, la superconductibilité, qui excitèrent vivement l'intérêt de 1913 à 1916, sont représentés par un grand nombre de mesures. Les nombreuses déterminations de susceptibilités magnétiques reflètent l'intérêt qui s'attache à la théorie du paramagnétisme, et les innombrables données, publiées sur la thermo-électricité et les effets galvano-magnétiques, témoignent du vif intérêt de ces questions dont l'importance croît de jour en jour.

Pour tous ceux qui s'occupent de l'électricité, du magnétisme ou de l'électrochimie, que ce soit au point de vue science pure, ou au point de vue de ses applications, cette collection unique de documents est absolument indispensable, non seulement à cause des chiffres eux-mêmes, mais à cause des bibliographies qui les accompagnent.

Pour faciliter la diffusion de cette documentation dans tous les milieux, le Comité de publication des Tables Annuelles a déc dé la publication sous cette forme de fascicules séparés, et il est certain que cette initiative sera hautement appréciée par les savants et les techniciens en faveur desquels elle a été prise.

Le prix de ces fascicules est d'ailleurs extrêmement modique, dans les conditions actuelles, et cette modicité voulue doit aider encore à la diffusion de cet ouvrage.

Il nous reste maintenant à souhaiter que le Comité, qui prépare en ce moment la publication des documents parus pendant les années 1917 à 1922, trouve les ressources nécessaires pour mener à Đien cette tâche considérable.
La Propriété scientifique. - Le projet de la (:. T. I. -.- Créalion d'un droil d'auteur pour le savant et l'inventeur, par Roger Dalmier et Louis Galdif. Préface de M. Emile Bomes, de l'Académie des Sciences. Prix 3 franes.

La propriété scientifique préocupe, et à juste litre, le moncle intellectuel. La Confédération des travailleurs industriels, cróe en mars 1920, et dont un des buts principaux est la défense et l'extension des droits des travailleurs intellectuels sur les produetions de leur travail, a entrepris des travaux qui ont abouli au texte d'une proposition de loi que précisćment MM. Dalimier et Gallić exposent en détail dans ce petit ouvrage.

Il comvient de dire que ce projet de loi a été présenté à la Commission de la Cooperation intellectuelle qui fort probablement ic soumettra à la Société des Nalions, avec un avis favorable.

\section{$*$}

Les Forces hydrauliques et les Usines hydroélectriques, … Aminagement des chutes d'eau et des centrales électriques, par E. P.coner, Ingénicur A. E. M., lauréat de Sociétés savantes et industrielles. - Delagrave, éditeur. - - En vente à la Librairie Rey, à Grenoble.

Le succès de ce petit volume est certain. En résumant les questions primordiales faisant l'objet d'un ouvrage beaucoup plus important du même auteur (1), il se propose de vulgariser les connaissances qui sont à la base de la science hydro-électrique.

Personne n'ignore l'importance que présente pour notre pays lutilisation de nos ressources naturelles, et en particulier de notre Houille Blanche. L'effort considérable fait depuis la guerre, les projets grandioses qui sont ou seront incessamment en voie de réalisation, font de laménagement de nos chutes d'eau une question d'actualité à laquelle personne ne saurait rester indifférent.

Aussi l'ouvrage de M. Pacoret répond-il à un réel besoin ; il s'adresse à tous, puisçu'il traite précisément la question de façon élémentaire, laissant de côté toutes les théories, tous les calculs que les spécialistes, qui seuls les recherchent, trouveront dans des ouvrages plus importants.

\section{$* *$}

Chaleur et Industrie vient de donner en supplément à son numéro d'avril une brochure intitulée : "La P'éparation des charbons" par M. Lomer, Ingénieur en chef des Mines.

Cette étude concernant d'une façon générale la préparation des charbons et particulièrement l'installation des ateliers de lavage, criblage et dépoussiérage, est accompagnée d'une annexe relative aux courbes de lavabilité et d'une partic descriptive donnanl le détail de diverses installations modernes de préparation des charbons (criblage de Lens, lavoir des Chavannes, rhéolaveurs de Marles, etc.).

Il s'agit done d'un importanl ensemble de documents dont l'intérêt, d'ailleurs, n'est pas pour les seuls spécialistes. Car le chropitre consacré au côlé commercial de la question, contient les plus utiles conseils pour tous les acheteurs el consommateurs de charbon. De sorte qu'il n'est aucun industriel qui ne doive s'y reporter avec fruit.

La brochure est en venle aux bureaux de la revue Chalcur $t$ Industrie, 5, rue Michel-Ange, Paris, all prix de 6 fr. 25 (port compris). 